STRUCTURAL SCIENCE CRYSTAL ENGINEERING MATERIALS

ISSN 2052-5206

Received 29 April 2019

Accepted 2 July 2019

Edited by A. J. Blake, University of Nottingham, England

Keywords: crystal nucleation; solution chemistry; solute-solvent interaction; desolvation; nucleation dynamics.

CCDC reference: 1906930

Supporting information: this article has supporting information at journals.iucr.org/b

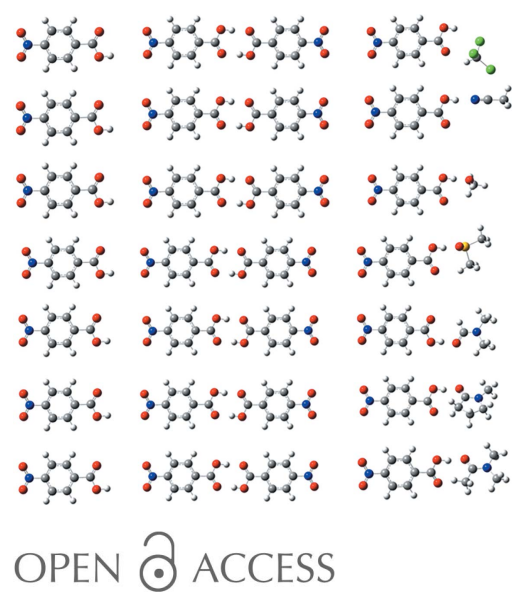

\section{Insight into the role of pre-assembly and desolva- tion in crystal nucleation: a case of $p$-nitrobenzoic acid}

\author{
Shuyi Zong, ${ }^{a}$ Jingkang Wang, ${ }^{\text {a,b }}$ Hao $\mathrm{Wu}^{\mathrm{a}}{ }^{\mathrm{a}} \mathrm{Qi}$ Liu, ${ }^{\mathrm{a}}$ Yunhui Hao, ${ }^{a}$ Xin Huang, ${ }^{\mathrm{a}, \mathrm{b} *}$ \\ Dehui $\mathrm{Wu}^{,}{ }^{\mathrm{c}}$ Guanchen $\mathrm{Zhou}^{\mathrm{c}}$ and Hongxun $\mathrm{Hao}^{\mathrm{a}, \mathrm{b}_{*}}$
}

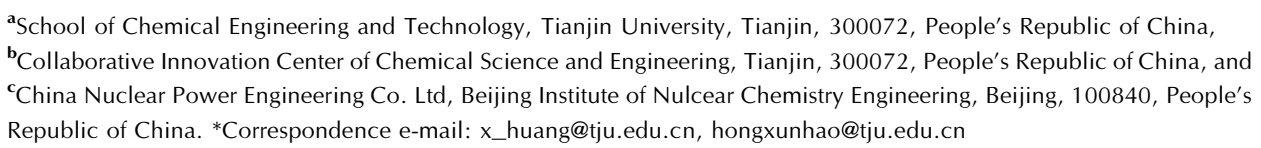

As one of the most important phenomena in crystallization, the crystal nucleation process has always been the focus of research. In this work, influences of pre-assembly species and the desolvation process on the crystal nucleation process were studied. $p$-Nitrobenzoic acid (PNBA) was taken as a model compound to investigate the relationship between solution chemistry and nucleation kinetics in seven different solvents. One unsolvated form and four solvates of PNBA were obtained and one of the solvates was newly discovered. The nucleation behaviours and nucleation kinetics of PNBA in the seven solvents were studied and analyzed. Density functional theory (DFT) and solvation energy calculation were adopted to evaluate the strength of solutesolvent interactions. Vibrational spectroscopy combined with molecular simulation was applied to reveal the pre-assembly species in the solution. Based on these results, a comprehensive understanding of the relationship between molecular structure, crystal structure, solution chemistry and nucleation dynamics was proposed and discussed. It was found that the structural similarity between solution chemistry and crystal structure, the interaction between specific sites and the overall strength of solvation will jointly affect the nucleation process.

\section{Introduction}

Nucleation is a key step in the crystallization process which has decisive influence on the crystal-size distribution and polymorphism of the final products. Unfortunately, although crystal nucleation from solution is common, there is insufficent understanding of the early stage in the crystal formation process and the nucleation mechanism is still not fully understood from the molecular level. In recent years, solution chemistry has turned out to be a useful tool to explore the molecular assembly path in the nucleation process due to the significant influence of solute-solvent interactions on the molecular self-assembly process (Du et al., 2015; Gebauer et al., 2014; Davey et al., 2013). Besides, the rapid development of computational techniques and the notable improvement of analysis techniques have made it possible to study larger molecular clusters more efficiently. Plenty of work therefore has been carried out to interpret the evolution of the so-called 'growth units' in the crystallization nucleation process (Gavezzotti et al., 1997; Chen \& Trout, 2008; Tommaso, 2013; Zeglinski et al., 2018). The structural correlation between solution aggregates and crystal syntheses has been investigated by studying the structural evolution process from solute 
molecules to supramolecular syntheses (Parveen et al., 2005; Byrn et al., 1976; Bernstein \& Hagler, 1978; Habgood, 2012).

It has been known that crystal nucleation is very sensitive to solution chemistry in the aspects of nucleation rate and nucleation polymorphism (Ostwald, 1897; Teychené \& Biscans, 2008). In recent years, many studies have shown that the structural arrangement of solute molecular clusters in solution will affect subsequent nucleation, and the synthons formed in solution will facilitate the nucleation and formation of the corresponding crystal structure (Cruz-Cabeza et al., 2017; Sullivan et al., 2014). The relationship between solution chemistry and nucleation was first discovered from 2,6-dihydroxybenzoic acid (Davey et al., 2001), and the direct evidence was first reported for tetrolic acid (Parveen et al., 2005). Subsequent spectroscopy studies supported the idea that the transformation from molecular self-assemblies to crystalline growth units was significantly affected by the solute-solvent interactions during the first stage of crystallization (clustering and nucleation), and these interactions could lead to the formation of supramolecular syntheses which could indirectly reflect the presence of synthons in crystalline structures (Hunter et al., 2012; Davey et al., 2006; Kulkarni et al., 2012; Mattei et al., 2013). The neutron scattering method was applied to carry out the structural analysis in detail. The results revealed the effects of solvents on the supersaturated state and confirmed the importance of solute desolvation in forming molecular clusters which would be further developed into nuclei and crystals (Burton et al., 2010).

In order to interpret these experimental phenomena and simulate crystal nucleation and growth processes, many efforts have been devoted to simulation techniques. Chen \& Trout (2008) used molecular dynamic simulation to analyze the influence of solvent on the solute synthon formation and concluded that the solute-solvent interactions played an important role in the self-assembly process of solute in solution. Khamar et al. (2014) have revealed the direct relationship between the strength of solute-solvent interaction and the experimental nucleation rates of salicylic acid via a modelling approach. The results indicated that the relative difficulty of nucleation was related to the strength of solvation and the associated difficulty of forming hydrogen-bonded dimers (Khamar et al., 2014; Yang et al., 2014). However, in most investigated systems, consistent molecular conformations in solution state and crystal state were due to the weak solvation effect. It is important to take strong solvation into account when exploring the nucleation transition states both experimentally and computationally.

In order to better understand the relationship between the molecular structure, crystal structure, solution chemistry and nucleation kinetics, investigations on the relationship between solution chemistry and nucleation kinetics were carried out using $p$-nitrobenzoic acid (PNBA) as the model compound. The nucleation process of PNBA in seven solvents [chloroform, acetonitrile, methanol, dimethyl sulfoxide (DMSO), $N, N$-dimethylformamide (DMF), $N$-methyl pyrrolidone (NMP), $N, N$-dimethylacetamide (DMA)] was investigated through a combination of spectroscopic techniques (NMR,
FTIR) and computational methods [including density functional theory (DFT), molecular dynamics and free-energy techniques]. The crystallization products (both pure crystal and solvates) and the self-association properties of PNBA in these seven solvents were studied in detail and the crystal nucleation rates were determined based on the induction time measurements at different supersaturations. The influences of solvent on the nucleation rates were studied in detail and the affecting mechanism of three key factors was proposed. Furthermore, computational chemistry was also adopted to form a self-consistent interpretation, which links solutesolvent interactions and molecular conformation to nucleation behaviours and crystallization products. More importantly, the formation of solvates was also taken into account in the study of the relationship between solution chemistry and nucleation kinetics.

\section{Experimental}

\subsection{Materials}

$p$-Nitrobenzoic acid (PNBA) was purchased from Aladdin Chemistry Co. Ltd, China, and its mass fraction purity was higher than $99 \%$. All selected solvents (chloroform, acetonitrile, methanol, DMSO, DMF, NMP, DMA) were analytical reagent grade with molar purity higher than $99.5 \%$ and were obtained from Tianjin Kewei Chemical Technology Co. Ltd, China. Chloroform- $d(99.8 \% \mathrm{D})$, acetonitrile- $\mathrm{d}_{3}(99.8 \% \mathrm{D})$, methanol- $d_{4}\left(99.8 \% \mathrm{D}\right.$, anhydrous), DMSO- $\mathrm{d}_{6}(99.5 \% \mathrm{D})$ and $\mathrm{DMF}^{-} \mathrm{d}_{7}(99.5 \% \mathrm{D})$ were purchased from Aladdin Reagent Co. Ltd of China. All chemicals were used without any further purification.

\subsection{Single crystal growth and determination}

The single crystals of PNBA form (I), (I).DMSO solvate, (I).DMF-solvate, (I).NMP-solvate and (I).DMA-solvate were cultivated using the slow solvent evaporation method. A saturated solution $(2 \mathrm{ml})$ of PNBA sealed with plastic film was placed into an oven and kept at $298.15 \mathrm{~K}$. Then, crystals of PNBA form (I) and solvates with suitable sizes for single crystal X-ray diffraction were collected after several days. The single-crystal data collection was conducted at $113 \mathrm{~K}$ on a Rigaku Rapid II diffractometer, Mo $K \alpha$ radiation $(\lambda=$ $0.71073 \AA$ ). Data collection and processing were performed with Rapid-auto (Rigaku/MSC, 2004). Data reduction and cell refinement were performed with SHELXS-97 (Sheldrick, 2008) and SHELXL-97 (Sheldrick, 2015), respectively.

\subsection{Computation methods}

Density functional theory (DFT) calculations were performed using the Gaussian 09 program to investigate interactions in (1:1) molecular complexes of PNBA in the seven solvents (Frisch et al., 2009). The equilibrium geometries of PNBA monomer, PNBA dimer, and 1:1 PNBA-solvent complexes were envisaged based on the single-crystal structure of PNBA form (I) and solvates. Then, these geometries were optimized by hybrid M06-2x function and $6-31+G(d, p)$ 
basis set with the Grimme D3 dispersion correction using SMD implicit solvation model (Grimme et al., 2010; Pratt et al., 2007). The Grimme dispersion correction allows a good description of weak interactions, such as van der Waals interactions. The binding energy ( $\Delta E_{\text {bind }}$ ) between two molecules is calculated using the following equation:

$$
\Delta E_{\text {bind }}=E_{\mathrm{AB}}-E_{\mathrm{A}}-E_{\mathrm{B}}+\mathrm{BSSE},
$$

where $E_{\mathrm{AB}}$ is the energy of the PNBA-solvent complex; $E_{\mathrm{A}}$ and $E_{\mathrm{B}}$ are the energies of the isolated monomers PNBA and solvent, respectively. All the energies have been corrected for the zero-point vibrational energies. BSSE is the basis set superposition error and calculated to correct the overestimation of binding energies due to the overlapping of basis functions (Boys \& Bernardi, 1970).

The solvation free energy was calculated by Materials Studio (version 7.0; Accelrys Software, 2013). The amorphous cell model composed of PNBA and solvent was chosen in the study, and each cubic periodic cell contained 1000 molecules. The Geometry Optimization simulation, MD simulation and Solvation Free Energy calculation were employed by Forcite module with COMPASS (Condensed-phase Optimized Molecular Potentials for Atomistic Simulation Studies) force field (Bunte \& Sun, 2000; Vyalov et al., 2017). Other parameters are given in Supporting Information.

\subsection{Spectroscopy analysis}

${ }^{1} \mathrm{H}$-NMR spectra were measured in chloroform because the solubility of PNBA in chloroform is too low to meet the concentration standard for ${ }^{13} \mathrm{C}-\mathrm{NMR}$ measurement. ${ }^{13} \mathrm{C}-\mathrm{NMR}$ spectra were measured in acetonitrile, methanol, DMSO and DMF, in which the solvents were representative. All ${ }^{1} \mathrm{H}-\mathrm{NMR}$ and ${ }^{13} \mathrm{C}$-NMR spectra experiments were conducted on a $500 \mathrm{MHz}$ liquid nuclear magnetic resonance spectrometer (Varian Inova $500 \mathrm{MHz}$ ) at $298 \mathrm{~K}$ after 32 and 256 scans, respectively. Data were processed and analyzed using MestReNova software. ${ }^{1} \mathrm{H}$ and ${ }^{13} \mathrm{C}$ chemical shifts were determined relative to an internal reference TMS. NMR chemical shifts calculations were performed using GaugeIndependent Atomic Orbital (GIAO) method as implemented in Gaussian09 (Cheeseman et al., 1996; Frisch et al., 2009). All the optimized monomer, dimer or complex structures were calculated using SMD implicit solvation model at the same M06-2x/6-31+G(d,p) level of theory (Pratt et al., 2007). The reported chemical shifts were relative to those of tetramethylsilane (TMS) calculated in the same way.

FTIR spectra results were recorded on an ATR-FTIR spectrometer (ReactIRTM45, Mettler-Toledo) equipped with Duradisc Dicomp probe for solution samples. For each sample, 32 scans were collected over spectra range from 800 to $4000 \mathrm{~cm}^{-1}$ at $2 \mathrm{~cm}^{-1}$ resolution to investigate the molecular structure of PNBA at different concentrations in the seven solvents tested.
Table 1

Crystallization products of PNBA in various solvents.

\begin{tabular}{ll}
\hline & Crystal form \\
\hline Chloroform & Form (I) \\
Acetonitrile & Form (I) \\
Methanol & Form (I) \\
DMSO & DMSO-solvate \\
DMF & DMF-solvate \\
NMP & NMP-solvate \\
DMA & DMA-solvate \\
\hline
\end{tabular}

\subsection{Nucleation kinetics study}

The spontaneous nucleation experiments of PNBA were performed in a round-bottomed jacketed glass batch crystallizer $(150 \mathrm{ml})$. The induction time, which is generally defined as the time period between the moments of establishment of constant supersaturation and formation of detectable crystal particles, was measured for PNBA using a turbidimeter (Crystal Eyes, DMS-2, HEL Ltd) with five to seven compositions in different solvents under $298.15 \mathrm{~K}$. The experimental apparatus is shown in Fig. S1. The experimental procedure is briefly described as follows: a certain amount of PNBA was added into the crystallizer together with different solvents and agitated with a mechanical stirrer at agitation speed of $300 \mathrm{rpm}$. The temperature was controlled by two thermostats (Julabo CF41, Germany) connected with two t-branch pipes. The temperature accuracy was $\pm 0.01 \mathrm{~K}$. The temperature was first set at $308.15 \mathrm{~K}$ for $1 \mathrm{~h}$ to clarify the solution. Then, the channels of t-branch pipes were changed to decrease the temperature to $298.15 \mathrm{~K}$. The moment when system temperature dropped to $298.15 \mathrm{~K}$ was used as the starting point of induction time and the end point was the moment when the turbidimeter indicated a sudden increase. Six reproducible experiments were performed at each composition to reduce the experimental error. The relative average deviation (RAD) was calculated by equation (2) to evaluate the accuracy of the data.

$$
\mathrm{RAD}=\frac{1}{N} \sum_{i=1}^{N}\left|\frac{t_{\mathrm{ind}, i}-\bar{t}_{\mathrm{ind}, i}}{\bar{t}_{\mathrm{ind}, i}}\right|
$$

where $N$ is the number of experimental measurements under the same condition; $t_{\text {ind, } i}$ and $\bar{t}_{\text {ind }, i}$ refer to the experimental induction time of experiment $i$ and the average value of reproducible experiments, respectively.

According to classical nucleation theory, the dependence of nucleation rate on supersaturation can be described by equations (3) and (4):

$$
\begin{gathered}
J=A S \exp \left(-\frac{B}{\ln ^{2} S}\right), \\
A=\frac{f_{\mathrm{o}} C_{0}}{\sqrt{12 \pi B}} B=\frac{16 \pi v_{0} \gamma^{3}}{3(k T)^{3}},
\end{gathered}
$$

where $J$ is the nucleation rate $\left(\mathrm{m}^{-3} \mathrm{~s}^{-1}\right), A$ is the nucleation kinetic parameter; $S$ is the degree of supersaturation, $B$ is the nucleation thermodynamic parameter, $f_{\mathrm{o}}$ is the attachment 
Table 2

Selected crystallographic data of form (I), DMSO-solvate, DMF-solvate, NMP-solvate and DMA-solvate of PNBA.

\begin{tabular}{llllll}
\hline & Form (I) & DMSO & DMF & NMP & DMA \\
\hline Empirical formula & $\mathrm{C}_{7} \mathrm{H}_{5} \mathrm{NO}_{4}$ & $\mathrm{C}_{9} \mathrm{H}_{11} \mathrm{NO}_{5} \mathrm{~S}$ & $\mathrm{C}_{10} \mathrm{H}_{12} \mathrm{~N}_{2} \mathrm{O}_{5}$ & $\mathrm{C}_{12} \mathrm{H}_{14} \mathrm{~N}_{2} \mathrm{O}_{5}$ & $\mathrm{C}_{11} \mathrm{H}_{14} \mathrm{~N}_{2} \mathrm{O}_{5}$ \\
Formula weight & 167.12 & 245.25 & 240.22 & 266.25 & 254.24 \\
Crystal system & Monoclinic & Monoclinic & Triclinic & Triclinic & Triclinic \\
Space group & $C 2 / c$ & $P 2{ }_{1} / c$ & $P \overline{1}$ & $P \overline{1}$ & $P \overline{1}$ \\
$a(\AA)$ & $21.136(4)$ & $22.714(5)$ & $6.2359(10)$ & $7.1402(4)$ & $7.2454(3)$ \\
$b(\AA)$ & $5.0489(10)$ & $7.6639(15)$ & $7.3281(2)$ & $7.5689(4)$ & $7.5178(3)$ \\
$c(\AA)$ & $12.904(3)$ & $13.026(3)$ & $12.5359(3)$ & $11.9955(5)$ & $11.9625(4)$ \\
$\alpha\left({ }^{\circ}\right)$ & 90 & 90 & $102.105(2)$ & $98.844(4)$ & $92.682(3)$ \\
$\beta\left({ }^{\circ}\right)$ & $97.00(3)$ & $104.68(3)$ & $99.957(2)$ & $102.693(4)$ & $107.121(3)$ \\
$\gamma\left({ }^{\circ}\right)$ & 90 & 90 & $91.211(2)$ & $100.077(5)$ & $105.088(3)$ \\
Volume $\left(\AA^{3}\right)$ & $1366.9(5)$ & $2193.5(9)$ & $550.70(2)$ & $610.09(6)$ & $595.85(4)$ \\
Density $\left(\mathrm{g} \mathrm{cm}^{-3}\right)$ & 1.624 & 1.485 & 1.449 & 1.449 & 1.417 \\
$Z$ & 8 & 8 & 2 & 2 & 2 \\
\hline
\end{tabular}

two DMSO molecules, while for DMF/ NMP/DMA-solvate, the asymmetric unit consists of one PNBA molecule and one solvent molecule, forming a PNBA-solvent heterodimer which was further stacked by face-to-face and/or face-to-side aromatic ring interactions.

According to Price et al. (2006), there are generally two main structural driving forces for the formation of solvates. (1) There are many voids in the packing arrangements of the main molecules, and the entrance of solvent can reduce the voids to improve the packing efficiency (Vippagunta et al., 2001). (2) The introduction of exotic solvents can form stronger and more stable intermolecular interactions than those involving

frequency of building units to a nucleus, $C_{0}$ is the concentration of nucleation sites, $v_{0}$ is the molecular volume $\left(\mathrm{m}^{3}\right), \gamma$ is the interfacial tension $\left(\mathrm{J} \mathrm{m}^{-2}\right), k$ is Boltzmann constant and $T$ is the absolute temperature $(\mathrm{K})$. By plotting the linear function of $\ln (J / S)$ versus $1 / \ln ^{2} S$, the pre-exponential kinetic factor $A$ can be derived from the intercept and the thermodynamic parameter $B$ can be obtained from the slope.

\section{Results and discussion}

\subsection{Crystallization outcomes and crystal structure data}

The solid forms of PNBA in chloroform, acetonitrile, methanol, DMSO, DMF, NMP and DMA at different temperatures and supersaturations were studied and the results are given in Table 1 . In chloroform, acetonitrile and methanol, the crystallization product is unsolvated polymorphic form (I). From single crystal XRD data, form (I) (CSD refcode NBZOAC 15) belongs to the monoclinic crystal system with the carboxylic acid $R_{2}^{2}(8)$ dimer, which is stacked through $\pi-\pi$ and $-\mathrm{CH} \cdots \pi$ interactions. However, in DMSO, DMF, NMP and DMA, the experiment results showed that PNBA crystallized as the corresponding solvates regardless of supersaturation or temperature. Although pure polymorphic form (I) is more thermodynamically stable (higher melting point) than solvates under atmospheric conditions, form (I) could transform into corresponding solvates in DMSO, DMF, NMP and DMA. Take DMSO for example, form (I) will transform to DMSO-solvate by solvent-mediated transformation in DMSO. The obtained single crystal data showed that DMSO-solvate, DMF-solvate and DMA-solvate were the same crystal forms with their CSD refcodes reported in literature as XIYGIY, XIYFUJ and XIYJEX, respectively (Dash et al., 2019). However, the single-crystal data of NMPsolvate was first solved and analyzed in this work. The details are listed in Table 2. Among them, DMSO-solvate belongs to monoclinic crystal system and the space group is $P 2_{1} / c$, while DMF-solvate, NMP-solvate and DMA-solvate all belong to triclinic crystal system with space group of $P \overline{1}$. The asymmetric unit of DMSO-solvate consists of two PNBA molecules and only the solutes (Tessler \& Goldberg, 2006). According to Table 2, DMSO-solvate, DMF-solvate, NMP-solvate and DMA-solvate all belong to the second case which can be explained by the carboxyl groups in PNBA which act as donors

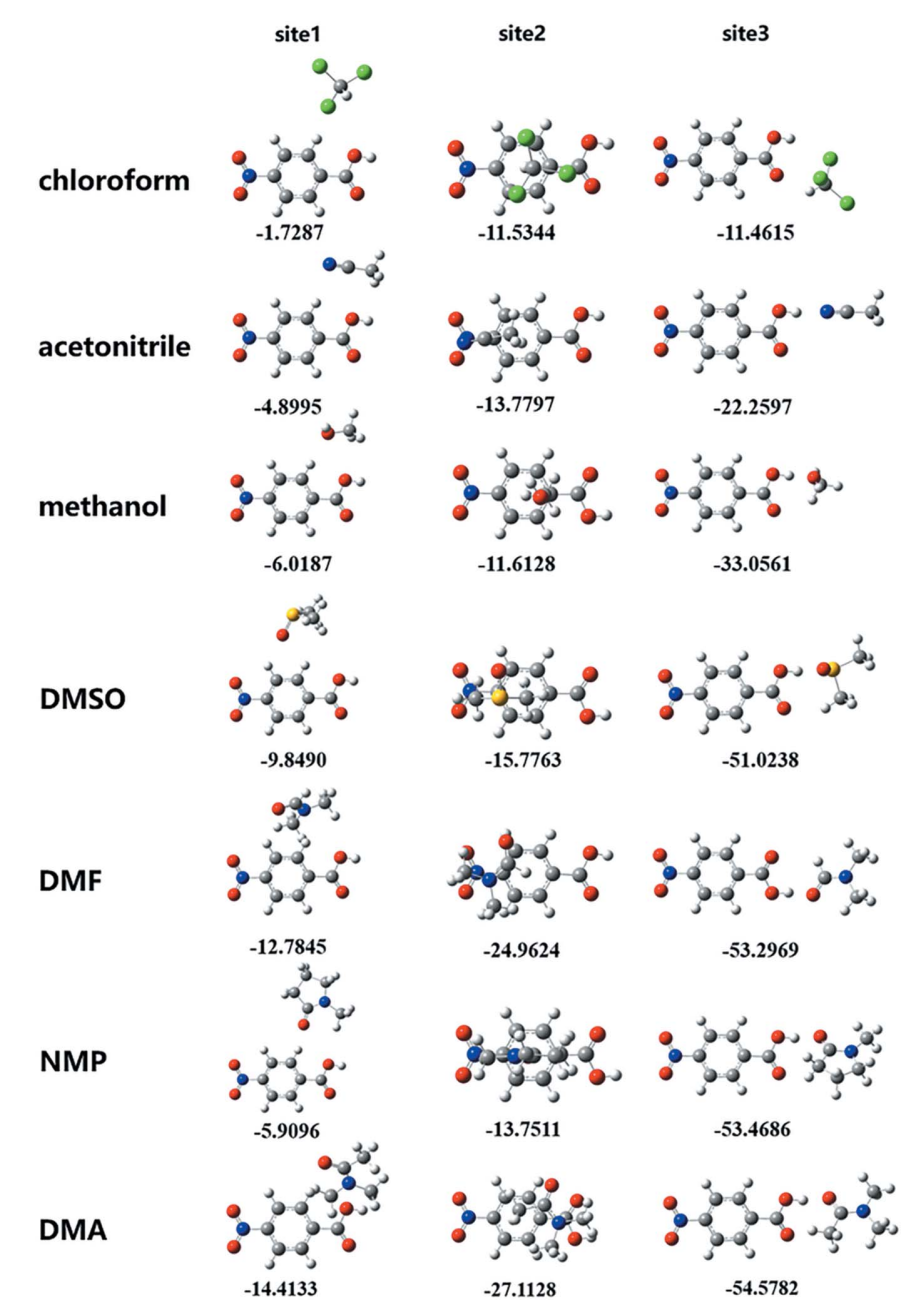

Figure 1

Optimized geometries and binding energies $\left(\mathrm{kJ} \mathrm{mol}^{-1}\right)$ for 1:1 PNBAsolvent complexes, calculated at M062X/6-31G(d,p) level. Carbon-grey, hydrogen-white, oxygen-red, nitrogen-blue, sulfur-yellow, chlorine-green. 
Table 3

Solvation free energy calculated by Material Studio 7.0.

\begin{tabular}{lcrrr}
\hline & $\begin{array}{l}\Delta G_{\text {id }} \\
\left(\mathrm{kJ} \mathrm{mol}^{-1}\right)\end{array}$ & $\begin{array}{l}\Delta G_{\text {vdw }} \\
\left(\mathrm{kJ} \mathrm{mol}^{-1}\right)\end{array}$ & $\begin{array}{l}\Delta G_{\text {elec }} \\
\left(\mathrm{kJ} \mathrm{mol}^{-1}\right)\end{array}$ & $\begin{array}{l}\Delta G_{\text {solv }} \\
\left(\mathrm{kJ} \mathrm{mol}^{-1}\right)\end{array}$ \\
\hline Chloroform & 50.70 & 34.19 & -264.18 & -179.31 \\
Acetonitrile & 90.00 & 166.07 & -1081.28 & -825.23 \\
Methanol & 505.65 & 373.40 & -3554.05 & -2674.97 \\
DMSO & 7311.32 & 2717.33 & -14367.87 & -4339.17 \\
DMF & 2650.79 & 849.59 & -11470.51 & -7970.13 \\
NMP & 8057.91 & 1868.21 & -28376.39 & -18450.22 \\
DMA & 9705.75 & -1645.46 & -31262.14 & -23201.84 \\
\hline
\end{tabular}

to form hydrogen bonds with solvents. Besides, the stronger the hydrogen-bond receptor capacity of the solvent is, the higher the solvation strength between the solute and the solvent will be, thus facilitating the self-assembly of solute and solvent molecules to form corresponding solvates. The calculated solute-solvent interactions in Section 3.2 also support this conclusion.

\subsection{Molecular interactions}

It is generally believed that electrostatic potential can be used to predict and explain the relative molecular orientation and the strength of the combination if a complex is mainly assembled by static electricity (such as hydrogen bond, dihydrogen bond, halogen bond, etc). And the more negative (or positive) the electrostatic potential is, the more electrophilic (nucleophilic) the atom is likely to be. Thus, the distribution of van der Waals surface electrostatic potential of molecules can be analyzed and used to predict the most active sites. According to the van der Waals surface electrostatic potential distribution diagram plotted by Multiwfn and VMD (Fig. S2) (Lu \& Chen, 2012a,b), three sites in PNBA were selected to optimize the 1:1 solute-solvent complexes and calculate the binding energy: benzene ring hydrogens (site 1), benzene ring $\pi$-electrons (site 2), and carboxyl hydrogen (site 3). The optimized geometries and binding energy results are shown in Fig. 1.

It can be seen from Fig. 1 that almost all the solute-solvent binding energies appear to be the weakest at site 1 and the strongest at site 3 . However, the binding energy of site 2 was a bit higher than that of site 3 in chloroform, and the binding energy of PNBA-chloroform is the weakest among all solvents for all the three sites. This is because acetonitrile, methanol, DMSO, DMF, NMP and DMA all have strong hydrogen-bond acceptors, favouring the formation of heterodimers which can significantly affect the binding energy. Besides, it can also be seen from Fig. 1 that the strongest solute-solvent binding at site 3 is observed for DMA, followed by NMP, DMF, DMSO, methanol, acetonitrile and chloroform. The four with the strongest binding (DMA, NMP, DMF and DMSO) also lead to the formation of corresponding solvates.

To obtain further insight into the interactions between PNBA and solvents, the total solvation free energies including short-range van der Waals forces and long-range electrostatic interactions were calculated. The calculated results are listed in Table 3 and the final configurations of simulation are shown
Table 4

Experimental and calculated chemical shifts for $\mathrm{H}_{16}, \mathrm{H}_{9}, \mathrm{H}_{5}, \mathrm{H}_{11}, \mathrm{H}_{3}, \mathrm{C}_{7}$, $\mathrm{C}_{6}, \mathrm{C}_{4}, \mathrm{C}_{2}$ and $\mathrm{C}_{1}$.

$a$ and $b$ are the empirical parameters of equation (5); $R^{2}$ is the fitting coefficient. The values of $a, b$ and $R^{2}$ are derived from linear regression by scaling method.

\begin{tabular}{|c|c|c|c|c|}
\hline & Experimental & $\begin{array}{l}\text { Computed } \\
\text { mono }\end{array}$ & $\begin{array}{l}\text { Computed } \\
\text { dimer }\end{array}$ & $\begin{array}{l}\text { Computed } \\
\text { solvated }\end{array}$ \\
\hline \multicolumn{5}{|c|}{ Chloroform } \\
\hline $\mathrm{H}_{16}$ & 11.17 & 8.63 & 13.44 & 8.71 \\
\hline $\mathrm{H}_{9}$ & 8.37 & 8.55 & 8.67 & 8.67 \\
\hline $\mathrm{H}_{5}$ & 8.35 & 8.46 & 8.63 & 8.60 \\
\hline $\mathrm{H}_{11}$ & 8.31 & 8.32 & 8.49 & 8.50 \\
\hline $\mathrm{H}_{3}$ & 8.27 & 7.73 & 8.47 & 8.29 \\
\hline$a$ & & 0.1353 & 1.7139 & 0.0718 \\
\hline$b$ & & 7.1344 & -5.7037 & 7.9152 \\
\hline$R^{2}$ & & 0.2304 & 0.9998 & 0.2972 \\
\hline \multicolumn{5}{|c|}{ Acetonitrile } \\
\hline $\mathrm{C}_{7}$ & 166.08 & 166.98 & 173.13 & 170.72 \\
\hline $\mathrm{C}_{6}$ & 151.71 & 155.72 & 157.10 & 154.49 \\
\hline $\mathrm{C}_{4}$ & 136.52 & 137.68 & 137.64 & 140.03 \\
\hline $\mathrm{C}_{2}$ & 131.79 & 135.75 & 135.75 & 135.60 \\
\hline $\mathrm{C}_{1}$ & 124.57 & 125.56 & 127.00 & 128.85 \\
\hline$a$ & & 0.9954 & 1.1174 & 1.0033 \\
\hline$b$ & & 2.8538 & -12.695 & 3.3344 \\
\hline$R^{2}$ & & 0.9905 & 0.9952 & 0.9982 \\
\hline \multicolumn{5}{|c|}{ methanol } \\
\hline $\mathrm{C}_{7}$ & 166.41 & 168.51 & 173.34 & 173.94 \\
\hline $\mathrm{C}_{6}$ & 150.76 & 166.16 & 157.37 & 154.33 \\
\hline $\mathrm{C}_{4}$ & 136.42 & 148.15 & 137.25 & 140.76 \\
\hline $\mathrm{C}_{2}$ & 130.76 & 145.96 & 136.23 & 135.84 \\
\hline $\mathrm{C}_{1}$ & 123.36 & 135.86 & 127.26 & 129.26 \\
\hline$a$ & & 0.7814 & 1.0843 & 1.0307 \\
\hline$b$ & & 42.324 & -7.1764 & 0.9435 \\
\hline$R^{2}$ & & 0.9204 & 0.9882 & 0.9935 \\
\hline \multicolumn{5}{|c|}{ DMSO } \\
\hline $\mathrm{C}_{7}$ & 175.30 & 167.20 & 173.11 & 173.04 \\
\hline $\mathrm{C}_{6}$ & 159.53 & 154.86 & 157.08 & 153.95 \\
\hline $\mathrm{C}_{4}$ & 145.85 & 138.61 & 137.62 & 142.08 \\
\hline $\mathrm{C}_{2}$ & 140.20 & 135.24 & 135.76 & 135.26 \\
\hline $\mathrm{C}_{1}$ & 133.25 & 127.95 & 127.00 & 128.48 \\
\hline$a$ & & 0.9483 & 1.1097 & 1.0458 \\
\hline$b$ & & 1.7562 & -21.26 & -11.177 \\
\hline$R^{2}$ & & 0.9936 & 0.9908 & 0.9965 \\
\hline \multicolumn{5}{|c|}{ DMF } \\
\hline $\mathrm{C}_{7}$ & 166.59 & 170.97 & 175.73 & 170.56 \\
\hline $\mathrm{C}_{6}$ & 151.09 & 155.64 & 155.85 & 153.95 \\
\hline $\mathrm{C}_{4}$ & 137.47 & 137.29 & 137.95 & 142.04 \\
\hline $\mathrm{C}_{2}$ & 131.51 & 135.79 & 136.28 & 135.32 \\
\hline $\mathrm{C}_{1}$ & 124.41 & 127.82 & 127.80 & 128.34 \\
\hline$a$ & & 1.0638 & 1.1342 & 0.9909 \\
\hline$b$ & & -1.938 & -14.579 & 5.1279 \\
\hline$R^{2}$ & & 0.9884 & 0.9873 & 0.9987 \\
\hline
\end{tabular}

in Fig. S3. DMA solvation has the strongest solute-solvent interaction, indicating the highest cohesive strength. Then, a trend of decrease in $\Delta G_{\text {solv }}$ can be seen from NMP, DMF, DMSO, methanol to acetonitrile. The weakest calculated strength was from chloroform, which is consistent with the weakest binding observed for the 1:1 PNBA-chloroform complex.

\subsection{Mechanism of molecular self-assembly}

NMR spectroscopy was used to further investigate the molecular interactions of PNBA in various solutions. The NMR chemical shifts can reflect not only the ensemble average interaction of the solution, but also the small changes 
in the local chemical environment of the molecule. The NMR chemical shifts of PNBA saturated solutions in chloroform, acetonitrile, methanol, DMSO and DMF were measured and the results are listed in Table 4. It was found that the NMR chemical shifts of low-concentration solutions were slightly different from those of high-concentration solutions in a certain solvent. However, it showed considerable difference (up to several ppms), especially for $\mathrm{H}_{16}$ and $\mathrm{C}_{7}$, in different solvents, revealing different solute-solvent interactions and different PNBA states.

In order to predict the form of PNBA existing in various solvents, the chemical shifts of PNBA monomer, dimer and solvated form in each solution were simulated by Guassian09 with SMD implicit solvation model (Frisch et al., 2009). All of the conformations were optimized or obtained from the corresponding single-crystal structures (Fig. S4). The calculated NMR chemical shifts for each conformation were linearly fitted to experimental values by scaling method. The equation is shown as follows:

$$
\delta_{\text {exp }}=\mathrm{a} \delta_{\mathrm{cal}}+b
$$

where $\delta_{\text {exp }}$ is the chemical shift measured by experiments; $\delta_{\text {cal }}$ is the chemical shift calculated by Gaussian09 (Frisch et al., 2009).

The obtained values of $a, b$ and the fitness $R^{2}$ are listed in Table 4. It is evident that the chemical shifts of carboxyl hydrogen $\mathrm{H}_{16}$ and carboxyl carbon $\mathrm{C}_{7}$ are more sensitive to the molecular conformation and the solvent medium. In chloroform, the fits between calculated and experimental values were excellent for dimers and unsatisfied for monomers and solvated form. Besides, the calculated chemical shift of $\mathrm{H}_{16}$ showed little difference from those of other protons in the chloroform-solvated form due to the low polarity of chloroform. The $\mathrm{H}_{16}$ chemical shift showed a considerable increase in dimer, indicating a significant deshielding effect, which is consistent with the experimental liquid NMR data. It can be concluded from these results that dimers were preferred over monomers and solvated forms in chloroform solution. However, the situation was different in acetonitrile, methanol, DMSO and DMF. In these solvents, the ${ }^{13} \mathrm{C}$ chemical shift fitted better for solvated form than monomers or dimers. The calculated $\mathrm{C}_{7}$ chemical shifts of dimers and solvated form in these solvents showed different degrees of increase compared
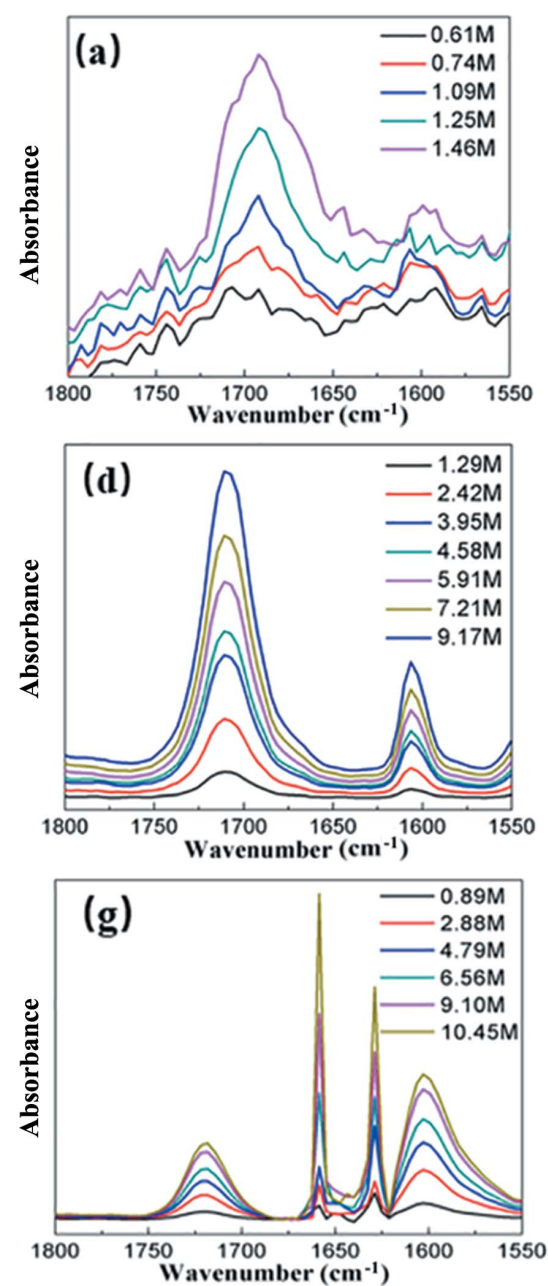
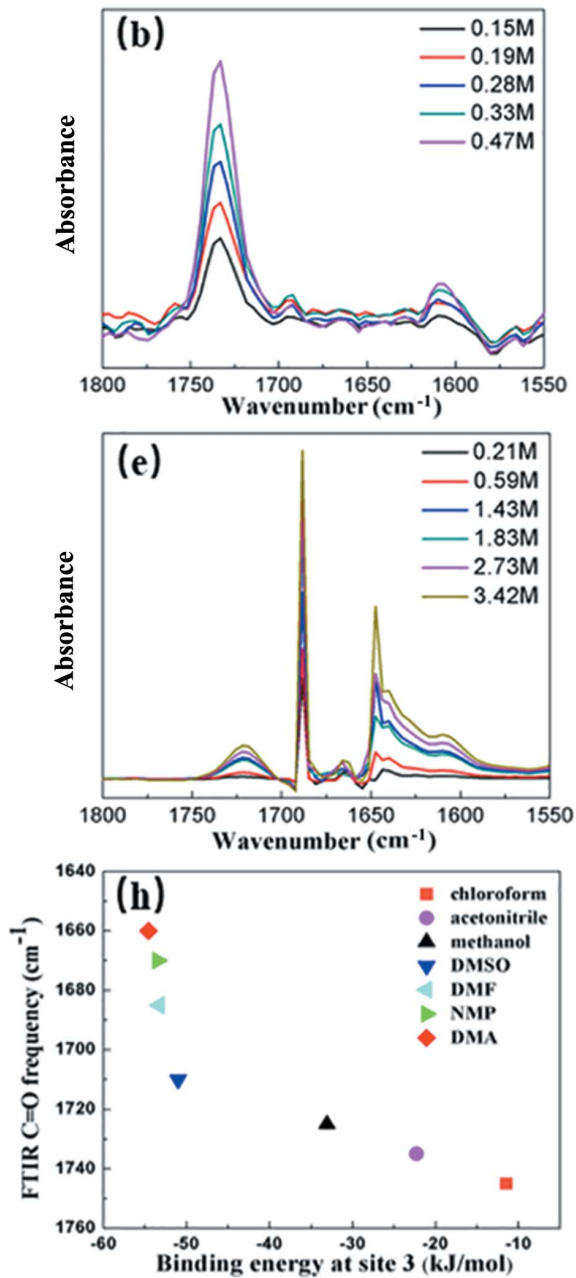
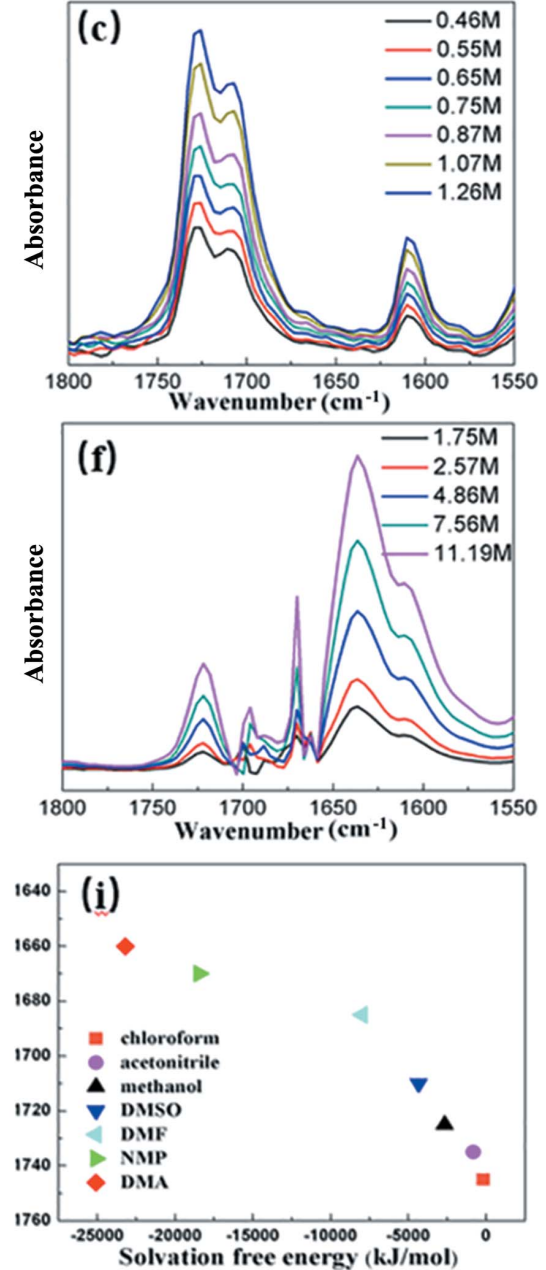

Figure 2

$(a)-(g)$ FTIR spectra of PNBA solutions in chloroform, acetonitrile, methanol, DMSO, DMF, NMP and DMA at different concentrations; $(h)$ relationship between carbonyl stretching and binding energy at site 3; (i) relationship between carbonyl stretching and solation free energy. 
to that of monomers. These differences were caused by the different intermolecular interactions of PNBA-solvent complexes and the different solvents polarity. The deshielding effect of solvated form could describe the experimental behaviours of liquid NMR.

In order to further confirm the form of PNBA existing in the selected solutions, FTIR spectroscopy was used to monitor the bands of $\mathrm{C}=\mathrm{O}$ stretching of PNBA in chloroform, acetonitrile, methanol, DMSO, DMF, NMP and DMA. The $\mathrm{C}=\mathrm{O}$ stretch peak is sensitive to carboxylic acid dimerization due to the hydrogen bond and the transition dipole coupling between two $\mathrm{C}=\mathrm{O}$ bonds in the dimer (Dybal et al., 1987). In general, the frequency of $\mathrm{C}=\mathrm{O}$ stretch of dimer is $40 \sim 50 \mathrm{~cm}^{-1}$ lower than that of monomer. The relative intensity of $\mathrm{C}=\mathrm{O}$ stretch is also strongly dependent on solution concentration: monomer peak dominates at low concentration while dimer peak dominates at high concentration. Thus, the $\mathrm{C}=\mathrm{O}$ stretch peaks of monomer and dimer will be visible in the mixture of monomers and dimers. In the FTIR spectra of PNBA in chloroform [Fig. 2(a)], two $\mathrm{C}=\mathrm{O}$ stretch peaks at $1745 \mathrm{~cm}^{-1}$ and $1695 \mathrm{~cm}^{-1}$ can be observed and the frequency splitting is $50 \mathrm{~cm}^{-1}$, which agrees with the usual difference of monomer and dimer in $\mathrm{C}=\mathrm{O}$ stretch frequencies (Fujii et al., 1988). Furthermore, it can be clearly seen that the relative intensities between the dimer band at $1695 \mathrm{~cm}^{-1}$ and the monomer band at $1745 \mathrm{~cm}^{-1}$ increased gradually as the solution concentration increased from $0.61 M$ to $1.46 M$, suggesting a tendency to form carboxylic acid dimers when increasing PNBA concentration in chloroform.

As for acetonitrile [Fig. 2(b)], there were two $\mathrm{C}=\mathrm{O}$ stretch peaks at $1735 \mathrm{~cm}^{-1}$ and $1695 \mathrm{~cm}^{-1}$. The carbonyl groups would not be solvated strongly in this case due to the absence of a hydrogen-bond donor in acetonitrile. Thus, due to weakly solvated carbonyl groups, the $1735 \mathrm{~cm}^{-1} \mathrm{C}=\mathrm{O}$ peak was only $10 \mathrm{~cm}^{-1}$ red shifted compared to that of monomer. The weak peak at $1695 \mathrm{~cm}^{-1}$ suggested the presence of a small amount of dimers in solution. Thus, the formation of dimers may be hindered by the solvation of carboxyl groups in acetonitrile. The intensity of $1735 \mathrm{~cm}^{-1}$ peak increased more significantly than the intensity of the $1695 \mathrm{~cm}^{-1}$ peak when concentration increased. The results further support the conclusion that solvated PNBA was favoured even in acetonitrile solution of high concentration.

However, PNBA showed different behaviours in methanol. As shown in Fig. 2(c), there were both a strong band at $1725 \mathrm{~cm}^{-1}$ and a weak shoulder band at $1705 \mathrm{~cm}^{-1}$ due to $\mathrm{C}=\mathrm{O}$ stretch. The frequency splitting was only $20 \mathrm{~cm}^{-1}$, much smaller than the typical value $\left(50 \mathrm{~cm}^{-1}\right)$ of carboxylic acid monomer-dimer equilibria. Besides, it can also be seen from Fig. 2(c) that the intensity ratio of the two peaks was completely independent of concentration. Thus, the two $\mathrm{C}=\mathrm{O}$ stretch peaks in methanol can not be derived from PNBA dimerization, even in supersaturated solution. It is most likely that the stronger peak at $1725 \mathrm{~cm}^{-1}$ was related to the solvated carbonyl group in methanol (formation of $\mathrm{C}=\mathrm{O} \cdots \mathrm{H}-\mathrm{O}$ hydrogen bond), and the weaker shoulder peak at $1705 \mathrm{~cm}^{-1}$ was the result of the weakly self-associated but not dimerized PNBA molecules. Similar phenomena have been observed for both $N$-methylacetamide in methanol and tolfenamic acid in ethanol, with exactly the same splitting frequency $\left(20 \mathrm{~cm}^{-1}\right)$ as PNBA in methanol (Du et al., 2015; Woutersen et al., 2001).

DMSO, DMF, NMP and DMA have strong hydrogen-bond acceptors and donors, and they can interact with both carboxyl groups and hydrogens of PNBA. The $\mathrm{C}=\mathrm{O}$ stretch peak of PNBA in DMSO solution was at $1710 \mathrm{~cm}^{-1}$ which was $35 \mathrm{~cm}^{-1}$ red shifted compared with that of monomer, and the $\mathrm{S}=\mathrm{O}$ stretch peak of DMSO at $1605 \mathrm{~cm}^{-1}$ was $20 \mathrm{~cm}^{-1}$ red shifted in comparison with that of pure DMSO solvent $\left(1625 \mathrm{~cm}^{-1}\right)$. These results indicated the formation of hydrogen bond in PNBA-DMSO solvate. Similar results were observed in DMF, NMP and DMA, in which the $\mathrm{C}=\mathrm{O}$ stretch peaks were at $1685 \mathrm{~cm}^{-1}, 1670 \mathrm{~cm}^{-1}$ and $1660 \mathrm{~cm}^{-1}$ respectively, indicating the formation of hydrogen bonds in corresponding solvates. Figs. 2( $h)$ and 2( $i)$ show the carbonyl stretch versus the binding

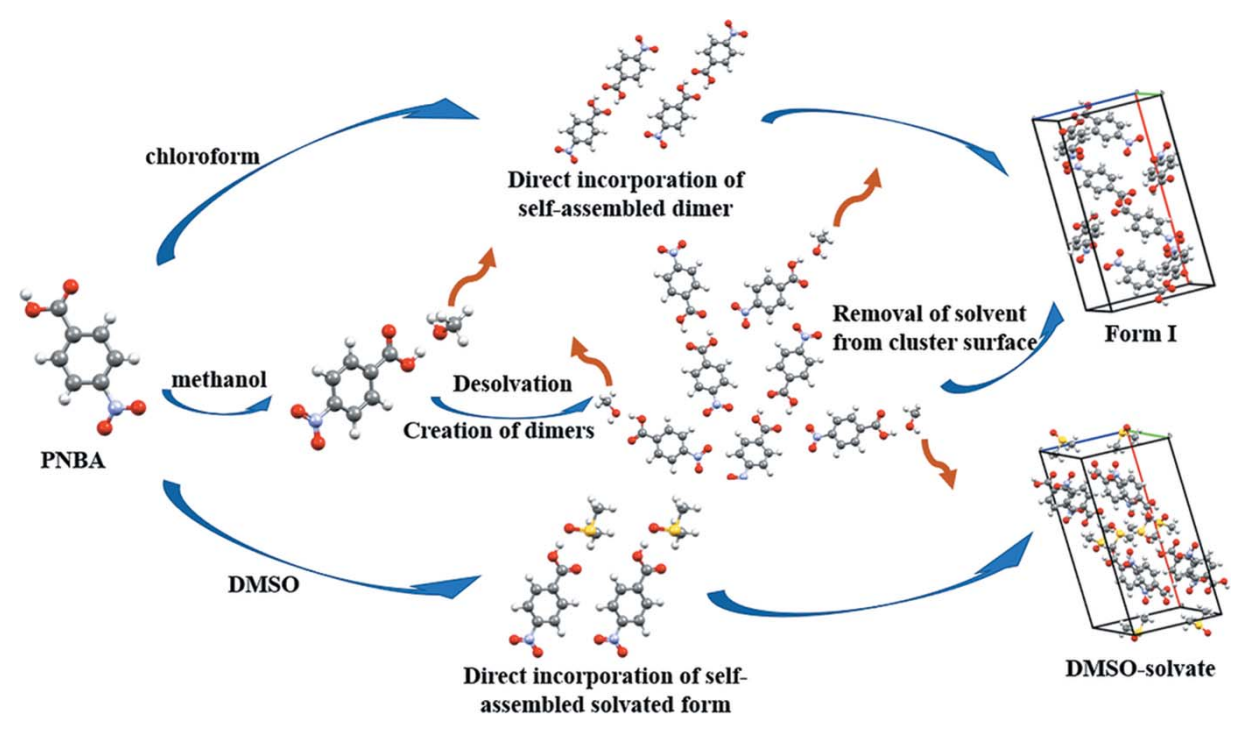

Figure 3

The proposed schematic diagram of molecular self-assembly mechanism during nucleation. 
Table 5

Summary of the classical nucleation theory (CNT) kinetic and thermodynamic parameters.

\begin{tabular}{lllll}
\hline & $A\left(\mathrm{~m}^{-3} \mathrm{~s}^{-1}\right)$ & $B\left(\times 10^{2}\right)$ & $f_{0} C_{0} / M\left(\times 10^{2}\right)$ & $\gamma\left(\mathrm{mJ} \mathrm{m}^{-2}\right)$ \\
\hline Chloroform & 16.51 & 0.10 & 30.02 & 0.50 \\
Acetonitrile & 13.97 & 0.42 & 2.50 & 0.79 \\
Methanol & 15.20 & 2.78 & 1.62 & 1.48 \\
DMSO & 11.63 & 0.32 & 0.22 & 0.52 \\
DMF & 20.82 & 0.74 & 1.35 & 0.66 \\
NMP & 22.92 & 0.94 & 1.06 & 0.69 \\
DMA & 17.64 & 1.07 & 0.64 & 0.71 \\
\hline
\end{tabular}

energy at site 3 and the solvation free energy, respectively. Based on the results, a conclusion can be drawn that stronger solute-solvent interaction will result in stronger deshielding effect of $\mathrm{C}=\mathrm{O}$ stretch peak.

The above conclusions from spectroscopy results indicated distinct solute-solvent interactions and self-assemblies of PNBA in the seven solvents. In chloroform, the computed results of dimers fitted best with that of NMR and two $\mathrm{C}=\mathrm{O}$ stretch peaks turned out to be concentration-dependent in FTIR analysis. These results jointly indicated that PNBA would form $R_{2}^{2}(8)$ carboxylic acid dimers in chloroform. However, PNBA showed different behaviours in acetonitrile, methanol, DMSO, DMF, NMP and DMA. $\mathrm{C}=\mathrm{O}$ stretch peaks had significant red shift, demonstrating that solute-solvent interactions would result in the formation of PNBA-solvent hydrogen-bonded complexes. Form (I) of PNBA was crystallized from chloroform, acetonitrile and methanol, while solvates of PNBA were obtained from corresponding solvents, including DMSO, DMF, NMP and DMA. The results could be explained by the following reasons: Firstly, the similarity between solution chemistry and crystal structure was significant when PNBA crystallized in chloroform, DMSO, DMF, NMP and DMA. Corresponding PNBA-PNBA homologous dimers or PNBA-solvent heterologous dimers are formed in solution and preserved in the final crystalline structures. However, when PNBA crystallized from acetonitrile and methanol, there was no direct correlation between solution chemistry and crystal structure. Although PNBA formed hydrogen bonds with the solvent molecules in solution, the final crystal structures did not contain solvent, ending up with PNBA-PNBA homologous dimers. The structural difference between solution chemistry and crystal synthesizer implies a unique nucleation pathway: the nucleation process contains an

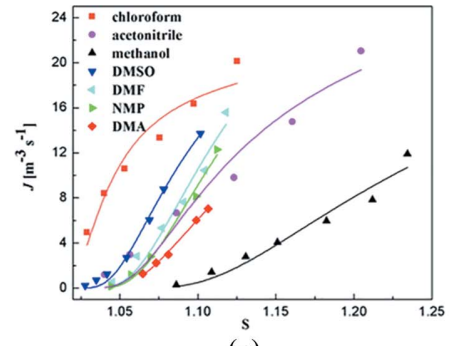

(a)

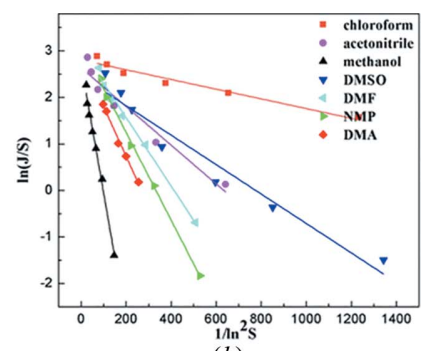

(b)
Figure 4

Relationship between (a) nucleation rate $J$ and supersaturation $S ;(b)$ $\ln (J / S)$ and $1 /\left(\ln ^{2} S\right)$. additional desolvation process prior to the formation of dimers (Fig. 3). The solvated PNBA aggregates undergo supramolecular recombination to remove the solvent and gradually form the dense crystal core containing only PNBAPNBA structures. Firstly, the clusters are likely to contain PNBA-solvent hydrogen-bonded complexes at the initial stage of nucleation. Then, they will be transformed into PNBAPNBA clusters through dissolvation. Finally, the stable crystal nuclei are eventually formed and they will continue to grow into bigger crystals.

\subsection{Mechanism of the nucleation process}

The induction time of PNBA in various solvents at different supersaturation was measured and the results are listed in Table S1. All the calculated RADs of reproducible experiments were less than $5 \%$, demonstrating a good reproducibility. The error was mainly caused by the inevitable time lag of the measurement technique. Thus, the fluctuation of measured induction time did not show the random phenomenon of induction time, which has commonly been observed in solutions of small volumes. The measured induction time therefore can be directly applied to estimate the nucleation rates and nucleation kinetic parameters of crystals (Kaschiev, 2000).

The crystal nucleation rates $J$ were plotted against solution supersaturation $S$ in Fig. 4(a). It revealed that, within the experimental supersaturation range, PNBA nucleation appeared relatively favourable in chloroform and became increasingly difficult in the order of DMSO, DMF, NMP, acetonitrile, DMA, and finally methanol. As shown in Fig. 4(b), $\ln (J / S)$ and $1 / \ln ^{2} S$ showed a good linear relationship in all seven solvents. The fitted parameters $A, B$, the calculated value of $f_{0} C_{0} / M$ (proportional to the attachment frequency) and interfacial energy $\gamma$ are listed in Table 5. It can be seen that the molecular attachment frequency followed the order of DMSO $<$ DMA $<$ NMP $<$ DMF $<$ methanol $<$ acetonitrile $<$ chloroform, which had no obvious relationship with that of nucleation rates data. In contrast, the order of interfacial energy $\gamma$ was chloroform $<$ DMSO $<$ DMF $<$ NMP $<$ DMA $<$ acetonitrile < methanol, almost the same with that of nucleation rates data. The exception of acetonitrile may be due to the effects of solvent's shape and volume on the nucleation of solvates. Thus, it can be inferred that the interfacial energy, which was closely related to the interactions between solute molecules and solvent molecules, played a crucial role in the nucleation rates of PNBA in these solvents.

Previous studies have revealed the relationship between nucleation rates and solute-solvent interactions. From the literature, three rules can be concluded as follows: (1) nucleation rates will increase when the conformational structure in solution is similar to the crystalline product (Petit et al., 1994); (2) the influences of solvents on nucleation may mainly come from interactions between specific sites rather than the overall solvation energy (Khamar et al., 2014); (3) nucleation rates will decrease when the binding between solvent and solute molecules become stronger (Zeglinski et al., 2018). 
Most results obtained in this work can be explained by these three rules. However, some results obtained in this work can not be completely interpreted by these rules since solvates, which are crystal-like but also strongly solvated, were taken into account in this work. As discussed above, the structures of PNBA in solutions of chloroform, DMSO, DMF, NMP and DMA were similar with those of PNBA in crystalline solids. However, different results were observed when additional dissolvation process were involved, such as in acetonitrile and methanol. According to the first rule, the nucleation rates of PNBA in chloroform, DMSO, DMF, NMP, DMA should be larger than those in acetonitrile and methanol. However, experimentally, PNBA nucleated more easily in acetonitrile than in DMA. Besides, both the strength of specific site interactions (site 3) and the overall solvation free-energy followed the trend of chloroform $<$ acetonitrile $<$ methanol $<$ DMSO $<$ DMF $<$ NMP $<$ DMA. Whereas, the order of nucleation rates was methanol $<$ DMA $<$ acetonitrile $<\mathrm{NMP}<$ DMF $<$ DMSO < chloroform. In DMSO, DMF, NMP and DMA systems, PNBA formed corresponding hydrogenbonded solvates which did not require complete desolvation, and the specific site structure (site 3) with the strongest interaction was retained in the final crystal structure. For these solvents, higher solvation free energy would result in slower nucleation rates: DMA $<$ NMP $<$ DMF $<$ DMSO. The reason is that PNBA nucleation in these solvents need to overcome the energy required for desolvation at other sites, and the growth of crystalline clusters to the critical nuclei also went through the desolvation process. In order to better understand the nucleation process, the environment of PNBA molecules and their interactions with surrounding molecules in these solvents were visualized by Multiwfn and VMD (Lu \& Chen, 2012a; Lu \& Chen, 2012b), as shown in Fig. 5. The red areas with the largest electron density represent the strongest interaction, such as hydrogen bond, while the blue areas with the least electron density do not have apparent interaction and the white areas with medium electron density indicate relatively weak interaction, such as $\pi-\pi$ stacking in these systems. It can be seen from Fig. 5 that, in the solution environment, in addition to the red areas at the carboxyl sites of PNBA, there were also relatively strong interactions at other sites which

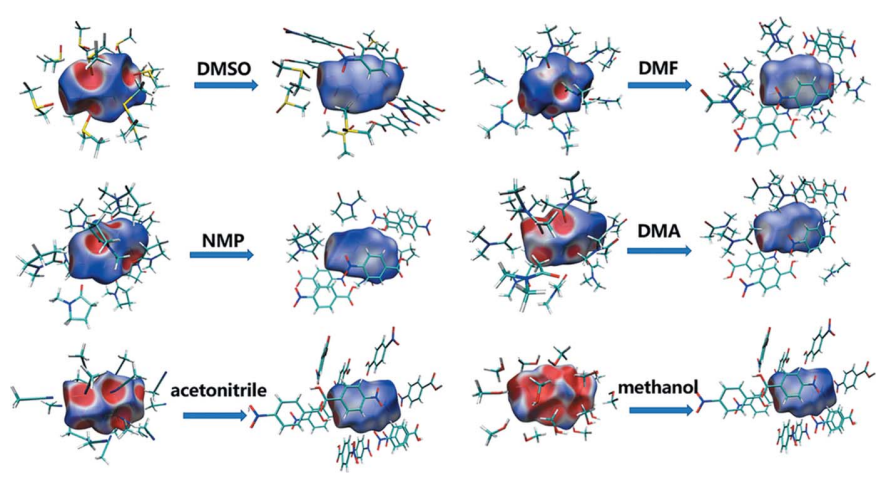

Figure 5

The environment of PNBA molecules and the interaction with surrounding molecules in various solvents and single crystals. transformed to relatively weak interactions when PNBA crystallized. Thus, apart from the interactions at carboxyl sites, PNBA molecules also had to overcome these interaction forces when they crystallized from liquid phase to solid phase. In other words, the solvation free energy can affect nucleation to some extent.

For solvents which did not form solvates, such as chloroform, acetonitrile and methanol, stronger binding between solvents and PNBA molecules in solution would result in more stable solute-solvent clusters and thus more energy would be required for desolvation process, slowing down the nucleation process. Due to the closest conformational structures in solution to the crystal structures in solid state, the nucleation in chloroform was the fastest since no desolvation process at carboxyl sites was needed. In addition, the smallest solvation free energy in chloroform indicated the relatively weak solute-solvent interactions at other sites. Thus, the aggregation of PNBA molecules in chloroform need to overcome weak interactions, which would also lead to faster nucleation rate, although the dimerization of PNBA in chloroform was also an effect of the weak solute-solvent interaction. For acetonitrile and methanol, the situation was also shown in Fig. 5. The solvated form at carboxyl sites in solution transformed to the carboxylic acid $R_{2}^{2}(8)$ dimers in the final crystal structures, and the stronger interactions (red areas) at other sites transformed to the weaker interactions (white areas). Thus, PNBA molecules had to overcome the overall solvation effects in acetonitrile and methanol, which leaded to the relatively low nucleation rates.

However, when all the solvent systems are considered together, no consensus relationship between the conformational structure similarity, the specific site interaction, the solvation free energy and the nucleation difficulty could be summarized. No single factor could individually describe the actual order of the nucleation difficulty and each factor does play a crucial role in certain situation. Therefore, it should be suggested that, none of the three factors: the similarity of the solute in liquid and solid states, the specific site interaction and the overall solvation free energy, could be neglected or underestimated. In fact, they jointly affect the crystal nucleation process.

\section{Conclusions}

In this work, investigations on the relationship between solution chemistry and nucleation kinetics were carried out by using $p$-nitrobenzoic acid (PNBA) as model compound. It was found that form (I) of PNBA could be obtained in chloroform, acetonitrile and methanol while corresponding solvates would be formed in DMSO, DMF, NMP and DMA. The crystal structures of all these forms were analyzed and discussed. NMR and FTIR spectroscopies were used to analyze the solute species in solution and the results showed that carboxylic acid dimers of PNBA were thermodynamically favoured in chloroform, whereas the solvated forms were favoured in acetonitrile, methanol, DMSO, DMF, NMP and DMA. The solute species in chloroform, DMSO, DMF, NMP and DMA 
were crystal-like while the conformation of solute in acetonitrile and methanol was unlike that in the crystal. In acetonitrile and methanol, one thermodynamically driven desolvation step was required to form the dense crystal nuclei containing only PNBA molecules.

Computational chemistry based on density functional theory (DFT) was used to calculate the intermolecular interactions and the results revealed that both the solute-solvent interactions at specific sites and the overall solvation free energy followed the order: chloroform < acetonitrile < methanol $<$ DMSO $<$ DMF $<$ NMP $<$ DMA. The nucleation kinetic results of PNBA showed an apparent solvent-dependent behaviour, with the nucleation difficulty in the order of chloroform $<$ DMSO $<$ DMF $<$ NMP $<$ acetonitrile $<$ DMA $<$ methanol. Based on the above results, it can be inferred that the structural similarity between solution chemistry and crystal structure, the interactions between specific sites and the overall solvation strength jointly affect the nucleation process. The results of this work confirm the importance of the pre-assembly and desolvation processes during the crystal nucleation. However, since the nucleation of crystals is complicated and many factors could affect the nucleation process, much more work needs to be carried out to fully understand the nucleation phenomenon.

\section{Funding information}

The following funding is acknowledged: National Natural Science Foundation of China (No. 21677081). The authors declare no competing financial interest.

\section{References}

Accelrys Software (2013). Materials Studio, release 7.0. Accelrys Software Inc., San Diego, CA, USA.

Bernstein, J. \& Hagler, A. T. (1978). J. Am. Chem. Soc. 100, 673681.

Boys, S. F. \& Bernardi, F. (1970). Mol. Phys. 19, 553-566.

Bunte, S. W. \& Sun, H. (2000). J. Phys. Chem. B, 104, 2477-2489.

Burton, R. C., Ferrari, E. S., Davey, R. J., Finney, J. L. \& Bowron, D. T. (2010). J. Phys. Chem. B, 114, 8807-8816.

Byrn, S. R., Graber, C. W. \& Midland, S. L. (1976). J. Org. Chem. 41, 2283-2288.

Cheeseman, J. R., Trucks, G. W., Keith, T. A. \& Frisch, M. J. (1996). J. Chem. Phys. 104, 5497-5509.

Chen, J. \& Trout, B. (2008). J. Phys. Chem. B, 112, 7794-7802.

Cruz-Cabeza, A. J., Davey, R. J., Sachithananthan, S. S., Smith, R., Tang, S. K., Vetter, T. \& Xiao, Y. (2017). Chem. Commun. 53, 79057908.

Dash, S. G., Singh, S. S. \& Thakur, T. S. (2019). Cryst. Growth Des. 19 , 952-958.

Davey, R. J., Blagden, N., Righini, S., Alison, H., Quayle, M. J. \& Fuller, S. (2001). Cryst. Growth Des. 1, 59-65.

Davey, R. J., Dent, G., Mughal, R. K. \& Parveen, S. (2006). Cryst. Growth Des. 6, 1788-1796.
Davey, R. J., Schroeder, S. L. \& ter Horst, J. H. (2013). Angew. Chem. Int. Ed. 52, 2166-2179.

Di Tommaso, D. (2013). CrystEngComm, 15, 6564-6577.

Du, W., Cruz-Cabeza, A., Woutersen, S., Davey, R. J. \& Yin, Q. (2015). Chem. Sci. 6, 3515-3524.

Dybal, J., Cheam, T. C. \& Krimm, S. (1987). J. Mol. Struct. 159, $183-$ 194.

Frisch, M. J. et al. (2009). Gaussian09, Revision D.01, Gaussian, Inc., Wallingford, CT, USA.

Fujii, Y., Yamada, H. \& Mizuta, M. (1988). J. Phys. Chem. 92, 67686772.

Gavezzotti, A., Filippini, G., Kroon, J., van Eijck, B. P. \& Klewinghaus, P. (1997). Chem. Eur. J. 3, 893-899.

Gebauer, D., Kellermeier, M., Gale, J. D., Bergström, L. \& Cölfen, H. (2014). Chem. Soc. Rev. 43, 2348-2371.

Grimme, S., Antony, J., Ehrlich, S. \& Krieg, H. A. (2010). J. Chem. Phys. 132, 154104.

Habgood, M. (2012). Phys. Chem. Chem. Phys. 14, 9195-9203.

Hunter, C. A., McCabe, J. F. \& Spitaleri, A. (2012). CrystEngComm, 14, 7115-7117.

Kashchiev, D. (2000). Nucleation: Basic Theory with Applications, pp. 1-30. Butterworth-Heinemann.

Khamar, D., Zeglinski, J., Mealey, D. \& Rasmuson, Å. C. (2014). J. Am. Chem. Soc. 136, 11664-11673.

Kulkarni, S. A., McGarrity, E. S., Meekes, H. \& ter Horst, J. H. (2012). Chem. Commun. 48, 4983-4985.

Lu, T. \& Chen, F. (2012a). Acta Phys. Chim. Sin. 28,1-18.

Lu, T. \& Chen, F. (2012b). J. Comput. Chem. 33, 580-592.

Mattei, A., Mei, X., Miller, A.-F. \& Li, T. (2013). Cryst. Growth Des. 13, 3303-3307.

Ostwald, W. Z. (1897). Z. Phys. Chem. 22, 289-330.

Parveen, S., Davey, R. J., Dent, G. \& Pritchard, R. G. (2005). Chem Commun. 12, 1531-1533.

Pratt, L. M., Truhlar, D. G., Cramer, C. J., Kass, S. R., Thompson, J. D. \& Xidos, J. D. (2007). J. Org. Chem. 72, 2962-2966.

Petit, S., Coquerel, G. \& Hartman, P. (1994). J. Cryst. Growth, 137, 585-594.

Price, C. P., Glick, G. D. \& Matzger, A. J. (2006). Angew. Chem. Int. Ed. 45, 2062-2066.

Rigaku/MSC (2004). Rapid-auto. Rigaku/MSC, The Woodlands, TX, USA.

Sheldrick, G. M. (2008). Acta Cryst. A64, 112-122.

Sheldrick, G. M. (2015). Acta Cryst. C71, 3-8.

Sullivan, R. A., Davey, R. J., Sadiq, G., Dent, G., Back, K. R., ter Horst, J. H., Toroz, D. \& Hammond, R. B. (2014). Cryst. Growth Des. 14, 2689-2696.

Tessler, L. \& Goldberg, I. (2006). J. Incl. Phenom. Macrocycl. Chem. 55, 255-261.

Teychené, S. \& Biscans, B. (2008). Cryst. Growth Des. 8, $1133-$ 1139.

Vippagunta, S. R., Brittain, H. G. \& Grant, D. J. W. (2001). Adv. Drug Deliv. Rev. 48, 3-26.

Vyalov, I., Vaksler, Y., Koverga, V., Miannay, F. A., Kiselev, M. \& Idrissi, A. (2017). J. Mol. Liq. 245, 97-102.

Woutersen, S., Mu, Y., Stock, G. \& Hamm, P. (2001). Chem. Phys. 266, 137-147.

Yang, H., Svärd, M., Zeglinski, J. \& Rasmuson, Å. C. (2014). Cryst. Growth Des. 14, 3890-3902.

Zeglinski, J., Kuhs, M., Khamar, D., Hegarty, A. C., Devi, R. K. \& Rasmuson, Å. C. (2018). Chem. Eur. J. 24, 4916-4926. 